\title{
Generator for single bubbles of controllable size
}

\author{
C. D. Ohla) \\ Department of Applied Physics, TU Twente, Postbus 217, 7500 AE Enschede, The Netherlands
}

(Received 6 June 2000; accepted for publication 9 October 2000)

\begin{abstract}
A new type of apparatus for the production of single bubbles of adjustable size is presented. A single bubble is generated by injecting a short burst of gas into a liquid channel flow. The radius of the bubble can be varied continuously from $300 \mu \mathrm{m}$ up to a $3 \mathrm{~mm}$ by adjusting the timing of a valve injecting the gas. The device works also in liquids with variable pressure and can be microcomputer controlled. (c) 2001 American Institute of Physics. [DOI: 10.1063/1.1329900]
\end{abstract}

\section{INTRODUCTION}

In many areas of science, e.g., the study of bubbly liquids, and technology, e.g., the aeration of liquid pools, gasliquid reactors and bubble-lift pumps, there is the need to produce bubbles of controllable sizes. Conventional needle type bubble generators ${ }^{1}$ have a severe drawback: they produce trains of bubbles with strong surface oscillations. Further, when they are applied in conditions of variable pressure, residual gas pockets in the needle might expand during a depressurization and disturb the measurements. More advanced needle injectors as used by $\mathrm{Kok}^{2}$ have the disadvantage of being costly and difficult to use in pressurized setup. The bubble generator presented by Kameda and Matsumoto ${ }^{3}$ which produces bubbles by "squeezing" air with a rubber plunger through a pin hole is able to generate small bubbles. However, the size of the bubble is mainly determined by the size of the pinhole and thus cannot be easily controlled.

In this new apparatus a single bubble is produced by injecting pressurized gas into a liquid channel flow. The channel flow is generated from a pressurized liquid reservoir connected with a valve to a cylindrical channel. After the flow has established a burst of pressurized air is injected. Near the tip of the injector tiny gas bubbles form and coalesce into a single bubble. This bubble is transported by the flow through the channel and an attached Plexiglas tubing towards a smooth expansion outlet of the channel where it is released into the main experimental container. The parameters which determine the bubble size are mainly the opening time of the gas injector and the pressure of the gas reservoir.

\section{DESCRIPTION OF APPARATUS}

Figure 1 sketches the bubble generator. The pressure for the gas $\left(P_{\text {gas }}\right)$ injection (upper valve in Fig. 1) is adjusted with a precision pressure controller (Buddenberg Ni-span $C$ tube). The lower valve for the channel flow is connected to a pressurized liquid reservoir at $\left(P_{\text {liquid }}\right)$ controlled with a simple manometer (Metalwork). We use air as the gas and highly purified water (filtered with Micropore) as the liquid medium. The two fast solenoid valves are common injection valves for gasoline engines (Bosch EV 1.3A). They have a

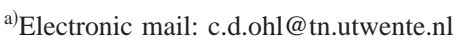

mechanical opening time of $1.5 \mathrm{~ms}$ and can work with difference pressures up to 3 bar. The valves fit onto a polyvinyl chloride (PVC) block containing the channel and are sealed with rubber $\mathrm{O}$ rings. The diameter of the channel and the Plexiglas tubing is $4 \mathrm{~mm}$. Large bubbles with diameters in the order of the tube diameter are in contact with the tubing while being transported by the liquid flow. The smooth expansion section for the release into the main working fluid helps to prevent a break up of these large bubbles. Additionally, by taking care that the surface of the channel and the transition between the channel and tubing are smooth one makes sure that these bubbles do not become trapped.

Figure 2 shows the electric circuit for driving one solenoid valve. A power metal-oxide-semiconductor fieldeffect transistor (MOSFET) connected to a capacitor delivers the current for opening the valve. The current is controlled

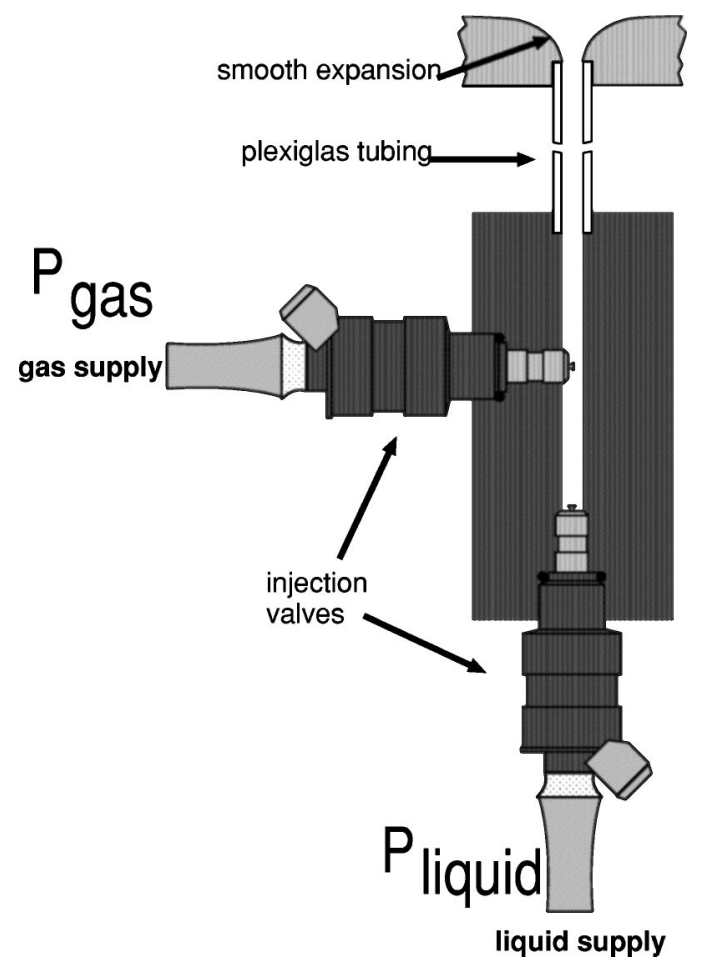

FIG. 1. Sketch of the apparatus, the lower valve applies a liquid stream from a pressurized reservoir, the upper one injects a short burst of pressurized gas. 


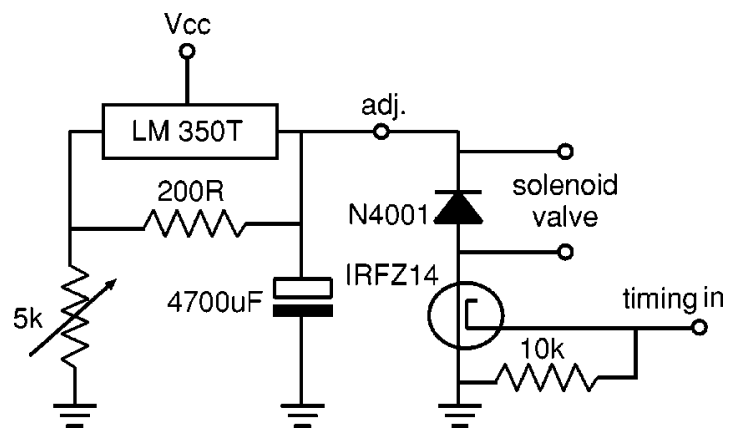

FIG. 2. Electric circuit of the MOSFET driver for one injection valve.

by adjusting the charging voltage of the capacitor with a voltage stabilizer (LM 350T) in conjunction with a potentiometer. To prevent reverse current, a diode is connected parallel to the solenoid. The timing of the valves is delivered with a microcontroller, having a serial line interface to receive the timing data from the lab computer (RxD), two digital outputs connected to the MOSFET and an interrupt line (INT) which serves as a trigger input (Fig. 3). The microcontroller is clocked with a crystal oscillator at $10 \mathrm{MHz}$. A program listing for a PIC16F84 (Microchip Corp.) can be found here. $^{4}$

Figure 4 depicts the timing diagram for the valves. After a trigger has been received the lower valves opens and generates a channel flow for $t_{1}+t_{2}+t_{3}$. Then after $t_{1}$ the gas supply valve opens for a time $t_{2}$. The bubble is being transported during the time $t_{3}$.

We now investigate the performance of the bubble generator working with air as the gaseous medium and water as the liquid medium. In this experiment, the pressure applied on the air valve is kept fixed to $P_{\text {gas }}=0.268$ bar and the water reservoir is pressurized to $P_{\text {liquid }}=0.9$ bar. The bubble generator is triggered and the individual bubbles are photographed after rising $1 \mathrm{~m}$ with a high speed video camera (Kodak EktaPro CR Imager 2000, 512×384 pixel having a single pixel resolution of $71 \mu \mathrm{m}$ ) at 250 frames per second. The exposure time is $300 \mu \mathrm{s}$. Depending on the bubble size 20-50 frames of the bubble with it present can be captured. These frames are transferred and processed on a lab computer. Here, the bubble shape is determined with a contour tracing algorithm from which the equivalent bubble radius

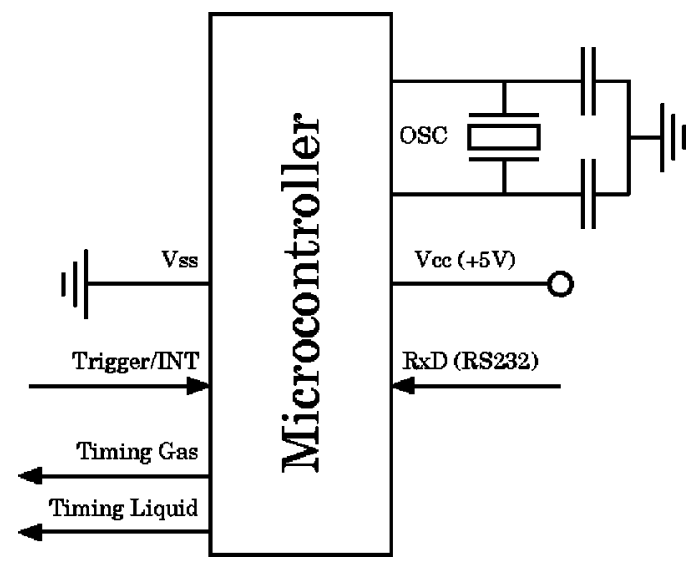

FIG. 3. Sketch of the microcontroller circuit for the timing.



FIG. 4. Timing diagram of the valves.

and the center of mass is obtained. By averaging over all frames of a sequence a mean bubble radius $R_{\text {mean }}$ is calculated.

In Figure 5 the results from multiple runs for five different opening times are summarized. The open symbols depict the average of $R_{\text {mean }}$ over ten runs, and the error bars their standard deviation. The standard deviation is about $10 \%$ of the bubble radius. However, for the largest bubbles measured, $R \approx 2.8 \mathrm{~mm}$, the standard deviation increases. These bubbles are more strongly deformed, compare Fig. 6, which makes it harder to obtain an equivalent bubble radius.

Additionally, in Fig. 5 a closed symbol is plotted for a different gas pressure, $P_{\text {gas }}=0.148$ bar, where bubbles of smaller size down to $R=0.4 \mathrm{~mm}$ but less reproducible can be generated. The smallest bubbles of radius down to $R$ $=0.33 \mathrm{~mm}$ have been found for $P_{\text {gas }}=0.122 \mathrm{bar}$, $P_{\text {liquid }}=0.6$ bar and $t_{2}=10 \mathrm{~ms}$.

With the described setup air bubbles in water with radii between 0.3 and $3 \mathrm{~mm}$ can be generated. The lower limit of $0.3 \mathrm{~mm}$ might be given by the dissolution time of the tiny

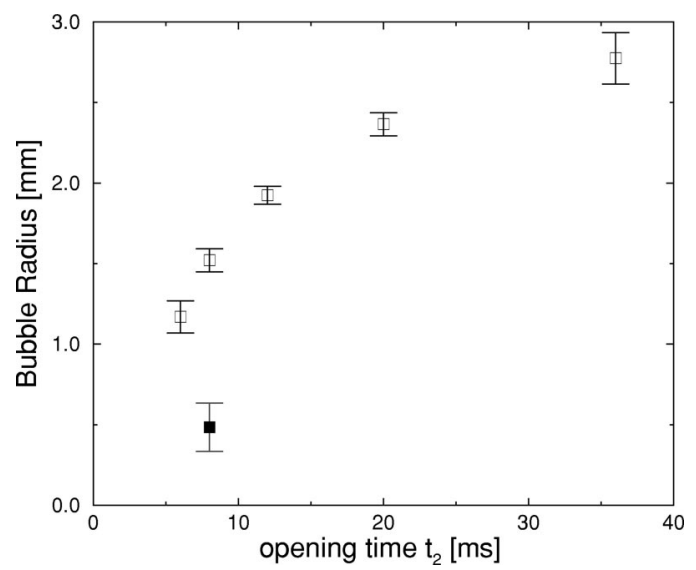

FIG. 5. Obtained bubble radius (air in water) vs opening time $t_{2}$ for $P_{\text {air }}=0.268$ bar (open symbols) and $P_{\text {air }}=0.148$ (closed symbol) plotted with the standard deviations over ten runs. The timings are $t_{1}=520 \mathrm{~ms}$ and $t_{3}$ $=3.1 \mathrm{~s}$. 


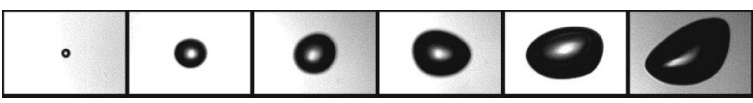

FIG. 6. Snapshots of bubbles generated with the device. The individual picture size is $8.5 \mathrm{~mm} \times 5.7 \mathrm{~mm}$.

bubbles and the upper limit is determined by the breakup due to surface oscillations when the bubble passes through the smooth expansion section. The author hopes that this device will be of value for others working in the field of multiphase flow.

\section{ACKNOWLEDGMENTS}

The author acknowledges the supply with valves from Robert Bosch GmbH Stuttgart, especially M. Voss. He also wants to thank the technicians G. W. Bruggert and H. Scholten for the construction work and discussions with A. Prosperetti. This research was supported in part by the European Union under Contract No. HPRN-CT-2000-00162.

${ }^{1}$ H. N. Oguz and A. Prosperetti, J. Fluid Mech. 257, 111 (1993).

${ }^{2}$ J. B. W. Kok, Eur. J. Mech. B/Fluids 4, 541 (1993).

${ }^{3}$ M. Kameda and Y. Matsumoto, J. Acoust. Soc. Am. 106, 3156 (1999).

${ }^{4}$ www.tn.utwente.nl/wsl/people/postdoc/dieter.html 